\title{
DEMANDA POR DOENÇAS CRÔNICO-DEGENERATIVAS ENTRE ADULTOS MATRICULADOS EM UMA UNIDADE BÁSICA DE SAÚDE EM SÃO CARLOS - SP
}

\author{
Adriana Barbieri Feliciano* \\ Suzana Alves de Moraes**
}

\begin{abstract}
FELICIANO, A.B.; MORAES, S.A.de. Demanda por doenças crônico-degenerativas entre adultos matriculados em uma unidade básica de saúde em São Carlos - SP. Rev.latino-am.enfermagem, Ribeirão Preto, v. 7, n. 3, p. 41-47, julho 1999.
\end{abstract}

O estudo teve por objetivo traçar o perfil da clientela maior de 12 anos de idade de uma UBS, segundo variáveis sóciodemográficas como sexo, faixa etária, cor, estado civil e procedência e caracterizar o perfil de morbidade segundo os capitulos da CID (X-Revisão) e grupos diagnósticos específicos destacando-se o grupo das doenças crônico-degenerativas, com vistas à sua caracterização no contexto da transição demográfico-epidemiológica. O delineamento foi do tipo transversal, sendo a população base constituída pela clientela matriculada na UBS até 31 de agosto de 1996. Foram excluídos os indivíduos em situação de abandono, os que foram transferidos para outras unidades e aqueles que foram a óbito. A população efetivamente estudada foi composta por 1013 indivíduos. Os dados foram processados no software FOXPRO vs 2.0 e as análises foram efetuadas no EPIINFO vs 6.04. Constatou-se que a população estudada foi basicamente constituída por mulheres (87.1\%). Com relação à idade $69.6 \%$ estavam entre 12 e 40 anos, $95.6 \%$ eram da cor branca, $57.2 \%$ eram casados e $97 \%$ procedentes da zona urbana. Quando os diagnósticos foram classificados segundo os capitulos da CID destacaram-se as doenças do aparelho geniturinário (35.5\%), aparelho respiratório (11.5\%) e sinais e sintomas (9,9\%). A classificação segundo os grupos especificos de causas apontou magnitude semelhante entre as doenças infecto-parasitárias (24.8\%) e as doenças crônico-degenerativas (24.5\%). No grupo de doenças crônico-degenerativas $50 \%$ dos capitulos da CID foram compostos pelo conjunto: causas circulatórias, endócrinas e mentais-comportamentais. Observou-se que o perfil de morbidade da UBS parece enquadrar-se no modelo polarizado de transição onde as doenças crônico-degenerativas coexistem com as infecto-parasitárias. O modelo tradicional de atendimento da Unidade que enfoca a saúde materno-infantil parece não adequar-se ao envelhecimento populacional progressivo ao priorizar uma clientela que embora ainda jovem já denota a necessidade de que sejam desenvolvidas ações coletivas voltadas para a prevenção primária e secundária das doenças crônico-degenerativas.

UNITERMOS: morbidade proporcional, doenças crônico-degenerativas, atenção primária a saúde

\section{INTRODUÇÃO}

Desde o início do século, as políticas de saúde no Brasil vêm se conformando no intuito de responder às necessidades exigidas para atender às relações de mercado. Assim, estas políticas evoluíram do modelo campanhista de saúde, até o modelo atual, com o predomínio da medicina privada (ALMEIDA s.d.; MENDES, 1994).

Este modelo de atenção centrado na doença não pode responder a algumas questões que tem sido colocadas hoje em toda a América Latina e no Brasil, principalmente a partir da segunda metade deste século, e decorrentes da transição demográfica e da transição epidemiológica.
Segundo LAURENTI (1990), a transição demográfica, ocorreu de maneira lenta e gradual nos países industrializados da Europa e EUA. No entanto, para o Brasil e países da América Latina, a queda acelerada da mortalidade a partir da década de 40, vêm contribuindo para a acumulação progressiva de idosos nestas populações. Em 1950, o Brasil ocupava o $16^{\circ}$ lugar em número de idosos (2,2 milhões); em 85 foi para o $11^{\circ}$ lugar (8,9 milhões) e em 2025 irá para o $6^{\circ}$ lugar com 33,8 milhões de idosos (KALACHE et al.,1987; RAMOS et al., 1987; VERAS et al., 1987; VERAS, 1988; BANCO MUNDIAL, 1991; RAMOS et al.,1993).

A transição demográfica traz intrinsicamente um outro conceito que é o de transição epidemiológica. Omran e outros autores descreveram este processo como

\footnotetext{
* Mestre em Enfermagem em Saúde Pública. Bolsista do CNPq de março de 1995 a março de 1997

** Doutora em Epidemiologia pela Faculdade de Saúde Pública da Universidade de São Paulo. Docente do Departamento de Enfermagem Materno-Infantil e Saúde Pública da Escola de Enfermagem de Ribeirão Preto da Universidade de São Paulo
} 
a evolução progressiva de um perfil de alta mortalidade por doenças infecciosas para um outro onde predominam os óbitos por doenças cardiovasculares, neoplasias, causas externas e outras doenças consideradas crônicodegenerativas (OMRAN, 1971, 1996; PRATA, 1992; BARRETO \& CARMO, 1995).

Embora a transição epidemiológica no Brasil esteja ocorrendo de maneira diferente em suas diferentes regiões, pode-se dizer que o aumento da mortalidade pelas doenças crônico-degenerativas vem sendo observado, principalmente, a partir da segunda metade do século. No Estado de São Paulo, a transição epidemiológica encontra-se em uma fase semelhante à dos países desenvolvidos onde existe um predomínio da morbimortalidade por doenças crônico-degenerativas quando comparadas com as doenças infecto-parasitárias. Em 1993, nos municípios de São Paulo e Ribeirão Preto, a mortalidade proporcional pelas doenças do aparelho circulatório neoplasias, causas externas e doenças do aparelho respiratório estavam entre as quatro primeiras causas de óbitos (PRATA, 1992; BARRETO \& CARMO, 1995; LAURENTI, 1990; RAMOS et al., 1987; INFORME EPIDEMIOLÓGICO DO SUS, 1992; SÃO PAULO. MP., 1993; RIBEIRÃO PRETO. SMS., 1993).

As políticas públicas de saúde a nível primário, ao priorizar a atenção a grupos populacionais específicos, como o grupo materno-infantil, não responde às necessidades decorrentes da transição demográficoepidemiológica que vem ocorrendo no Brasil e América Latina a partir da segunda metade deste século.

Com o propósito de investigar a adequação de uma Unidade Básica de Saúde (UBS) para o atendimento de uma demanda supostamente decorrente do processo de transição o estudo teve por objetivos identificar características sócio-demográficas e o perfil de morbidade dos adultos matriculados na UBS, procurando-se detectar, neste ambiente, a situação das doenças crônicodegenerativas.

\section{MATERIAL E MÉTODOS}

\subsection{Delineamento do estudo}

O estudo se caracterizou como sendo do tipo descritivo de corte transversal.

\subsection{População estudada}

Excluídos os indivíduos em situação de abandono, os que foram transferidos e os que foram a óbito a população efetivamente estudada foi constituída por 1013 indivíduos, acima de 12 anos, matriculados e freqüentes na unidade, no período de agosto de 1995 a agosto de 1996.

\subsection{Local e período de coleta dos dados}

O estudo foi realizado na Unidade Básica de Saúde Dr Romeu de Cresce (São Carlos-S.P.) e a coleta dos dados foi procedida no segundo semestre de 1996.

\subsection{Procedimentos utilizados para a coleta dos dados}

Os dados de identificação (sexo, cor, estado civil, procedência, faixa etária) e diagnósticos, foram coletados dos prontuários dos indivíduos em fichas do tipo catálogo (tamanho 5x7). Após a coleta dos dados de identificação, foram coletados todos os diagnósticos dos indivíduos do estudo.

\subsection{Caracterização diagnóstica}

Todas as doenças foram classificadas segundo os grandes grupos de causas conforme a Classificação Internacional de Doenças - CID-X Revisão. (OMS, 1995). Este processo se deu após a coleta dos dados, onde para cada diagnóstico ou queixa, encontrados nos prontuários, foi atribuído o seu código correspondente da CID-X Revisão.

Pela dificuldade de se estabelecer uma hierarquia diagnóstica com base nos dados dos prontuários da Unidade Básica de Saúde, foram necessários alguns ajustes nos bancos de dados, a fim de que os diagnósticos fossem reduzidos a dois principais, de maneira que se pudesse capturar as doenças crônicas em meio a uma tão grande diversidade diagnóstica. Deste modo, quando o indivíduo apresentava algum diagnóstico de etiologia crônica, foram eliminadas as informações referentes às queixas relatadas. Foram eliminados os diagnósticos de etiologia infecciosa e duração rápida, como também, as causas não crônicas de etiologia ginecológica. Quando o indivíduo apresentava vários diagnósticos do mesmo grande grupo de causas, permaneceu o de maior relevância no grupo.

\subsection{Processamento dos dados}

Após a codificação dos dados em fichas de catálogo, os mesmos foram transpostos para uma planilha formatada no programa FOX PRO vs 2.0 para microcomputador (FOX HOLDINGS INC, 1991).

Durante a fase de processamento de dados optouse por trabalhar com três novas variáveis relacionadas ao diagnóstico:

1- Variável diagnóstico - esta foi reduzida a dois principais 
não obedecendo a nenhum critério do tipo principal e/ou secundário;

2- Variável CID - atribuiu a cada diagnóstico o respectivo capítulo a que pertencia na CID-X;

3- Variável GRUPO - atribuiu a variável "diagnóstico" uma classificação de acordo com os grupos especificados a seguir: CRON (para as doenças crônicas), INFE (para as infecto-parasitárias), GO (para as de etiologia ginecológica e obstétrica), SISEX (para os sinais e sintomas), PROSERV (para as situações de procura por serviços de saúde), CEX (para as causas externas), OUT para todas as outras doenças não classificadas nos grupos acima.

Com o propósito de tornar possível a caracterização da morbidade segundo a variável "Diagnóstico" utilizou-se um recurso do programa FOX PRO chamado "APEND" que permitiu transformar as variáveis "DIAGNÓSTICO 1 e 2 ", “CID 1 e 2 " e "GRUPO 1 e 2" nas respectivas variáveis chamadas "DIAGNÓSTICO”, “CID” e "GRUPO”, permanecendo ao final um banco de dados com um total de 2026 registros para posterior análise.

\subsection{Análise dos dados}

O processo de análise dos dados foi realizado em duas etapas:

Etapa 1- Nesta fase pretendeu-se uma caracterização dos indivíduos da população estudada enquanto indivíduos. Para tanto foram utilizadas as variáveis sóciodemográficas sexo, idade, procedência, estado civil e cor. Etapa 2- Em uma fase posterior, pretendeu-se a caracterização do perfil de morbidade, principalmente pelas doenças crônico-degenerativas, utilizando-se indicadores de morbidade proporcional. Nesta fase utilizou-se os dados de morbidade enquanto diagnósticos e não enquanto indivíduos, verificando-se também este perfil segundo as variáveis sócio-demográficas.

A análise dos dados foi efetuada no módulo "Analysis" do Programa EPIINFO vs 6.04 para microcomputador (DEAN, 1996).

\subsection{Considerações éticas}

A consulta aos prontuários da UBS para obtenção dos dados foi permitida através de consentimento formal por uma carta ofício, encaminhada ao Secretário Municipal de Saúde do município de São Carlos que autorizou a manipulação dos mesmos. Os nomes dos pacientes foram tratados pelas letras iniciais e os diagnósticos pelos códigos da CID-10, mantendo-se preservado sigilo absoluto quanto às informações neles contidas.

\section{RESULTADOS}

Observou-se que tem feito parte do contingente populacional desta unidade uma grande maioria de mulheres $(87.1 \%)$, em relação aos indivíduos do sexo masculino $(12.9 \%)$. Os dados apontaram para uma população constituída em sua maioria por indivíduos da cor branca $(95.6 \%)$ e que também grande parte era proveniente da zona urbana (97\%).

Ocorreu um predomínio dos indivíduos nas faixas etárias mais jovens, menores de 40 anos $(69.6 \%)$, quando comparada com a população acima de 60 anos (9.1\%). Com relação ao estado civil $57.2 \%$ eram casados e $36.1 \%$ eram solteiros.

Foi verificado para esta unidade um índice de razão de dependência de $13.82 \%$, ou seja, para cada 100 indivíduos pertencentes à população economicamente ativa cerca de 14 indivíduos eram dependentes economicamente.

Ao se levar em consideração a data para a procura de matrícula na unidade observou-se que o período de maior procura foram os meses de março a junho (Figura 1).

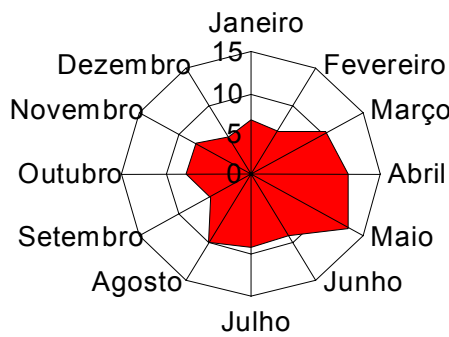

Figura 1 - Distribuição percentual dos indivíduos segundo o mês de procura para matrícula na unidade

Com relação à classificação segundo os grandes capítulos da CID-X, observou-se que existe um predomínio das doenças do aparelho geniturinário (35.5\%), as doenças infecto-parasitárias estão em quarto lugar $(9.3 \%)$ e as doenças do aparelho circulatório aparecem em quinto lugar (8.3\%) (Figura 2).
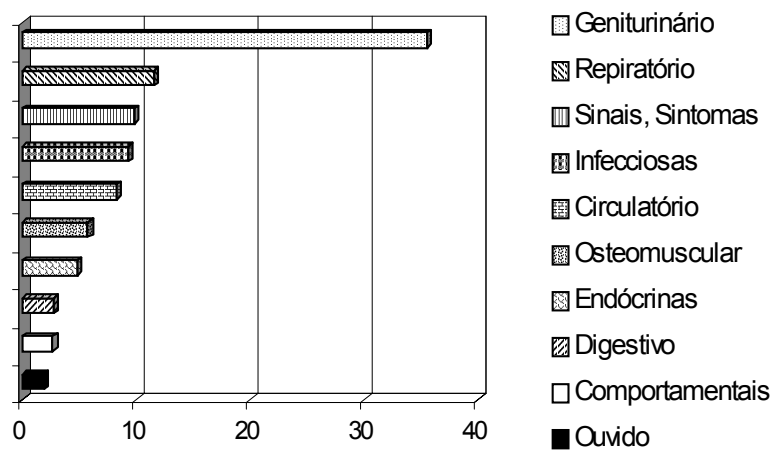

Figura 2 - Distribuição percentual dos dez principais capítulos da CID-X 
A caracterização da morbidade segundo a variável "diagnóstico" encontra-se na Tabela 1.

Tabela 1 - Número e porcentagem de diagnósticos mais freqüentes

\begin{tabular}{lcc}
\hline \multicolumn{1}{c}{ Principais Diagnósticos } & $\mathrm{N}^{\mathbf{0}}$ & $\mathbf{\%}$ \\
\hline Causas ginecológicas e obstétricas & 494 & 31,2 \\
Hipertensão arterial & 99 & 6,3 \\
Resfriado comum & 60 & 3,8 \\
Infecção urinária de loc alização não & 48 & 3,0 \\
especificada & 43 & 2,7 \\
Parasitose intestinal não especificada & 42 & 2,7 \\
Amigdalite aguda & 35 & 2,2 \\
Diabetes mellitus não especificado & 26 & 1,6 \\
Dor lombar baixa & 22 & 1,4 \\
Bronquite não especificada como aguda ou \\
crônica & 17 & 1,1 \\
Gastrite e duodenite & 695 & 44 \\
Outros & $\mathbf{1 5 8 1}$ & $\mathbf{1 0 0}$ \\
\hline ToTAL & & \\
\hline
\end{tabular}

Ao excluírem-se as causas ginecológicas, o principal diagnóstico foi o de hipertensão arterial (6.3\%), seguido por quatro diagnósticos relacionados às causas infecto-parasitárias (12.2\%) e mais quatro diagnósticos relacionados às doenças crônicas (12.6\%).

Ao se verificar a morbidade segundo a variável "Grupo" detectou-se que as doenças infecto-parasitárias corresponderam a $24.8 \%$ e as crônico-degenerativas a $24.5 \%$.

No grupo de doenças infecto-parasitárias o diagnóstico mais comum foi o resfriado comum (15.4\%), seguido da infecção do trato urinário não localizado $(12.3 \%)$, cabendo às verminoses não definidas o terceiro lugar deste grupo como causa de morbidade. Neste grupo existe um predomínio das doenças do aparelho respiratório (30.5\%) (Tabela2).

Tabela 2 - Número e porcentagem de diagnósticos mais freqüentes no grupo de doenças infecto-parasitárias

\begin{tabular}{lcc}
\hline \multicolumn{1}{r}{ Diagnósticos } & $\mathbf{N}^{\mathbf{0}}$ & $\mathbf{\%}$ \\
\hline Resfriado comum & 60 & 15,4 \\
Infecção do trato urinário & 48 & 12,3 \\
Parasitose intestinal & 43 & 10,9 \\
Annigdalite aguda & 42 & 10,8 \\
Diarréia & 25 & 6,3 \\
Sinusite aguda & 17 & 4,3 \\
Outras & 157 & 40,0 \\
\hline TOTAL & $\mathbf{3 9 2}$ & $\mathbf{1 0 0}$ \\
\hline
\end{tabular}

No grupo de doenças crônicas o principal diagnóstico foi a hipertensão arterial (25.5\%), seguida do diabetes mellitus (9.0\%). Observa-se que neste grupo existe uma diversidade diagnóstica, onde cada diagnóstico pertence a um capítulo diferente da CID-X (Tabela 3).

Tabela 3 - Número e porcentagem de diagnósticos mais freqüentes no grupo de doenças crônicas

\begin{tabular}{lcc}
\hline \multicolumn{1}{c}{ Diagnósticos } & $\mathbf{N}^{\mathbf{0}}$ & $\mathbf{0}$ \\
\hline Hipertensão arterial & 99 & 25,5 \\
Diabetes mellitus não especificado & 35 & 9,0 \\
Anemia por deficiência de ferro & 29 & 7,5 \\
Bronquite & 22 & 5,7 \\
Transtomo neurovegetativo & 20 & 5,2 \\
Gastrite e duodenite & 17 & 4,4 \\
Outras & 161 & 43,4 \\
\hline \multirow{2}{*}{ TOTAL } & $\mathbf{3 8 8}$ & $\mathbf{1 0 0}$ \\
\hline
\end{tabular}

Os três principais capítulos da CID-X para o grupo de doenças crônicas foram: 1- Doenças do aparelho circulatório; 2- Doenças endócrino-metabólicas; 3Doenças mentais-comportamentais. (Figura 3).

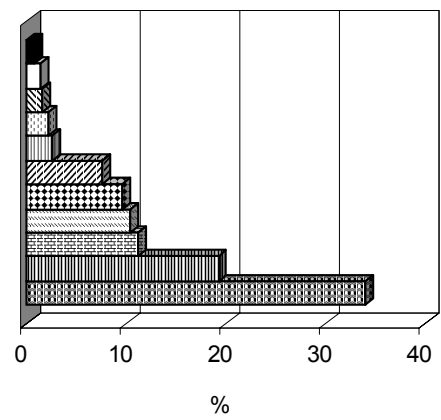

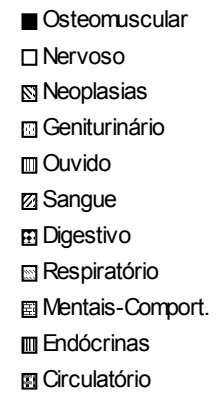

Figura 3 - Distribuição percentual de capítulos da CID-X no grupo de doenças crônicas

Entre as causas circulatórias as principais doenças foram hipertensão arterial $(76 \%)$, febre reumática $(5 \%)$, arritmia (4\%), varizes (3\%) (Figura 4).

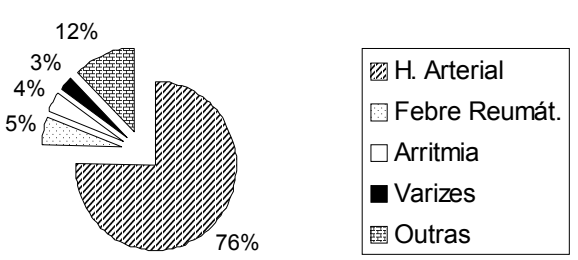

Figura 4 - Distribuição percentual dos diagnósticos mais freqüentes entre as causas circulatórias 
Entre as causas endócrino-metabólicas as principais doenças foram o diabetes mellitus $(46.1 \%)$, hipercolesterolemia (22.4\%), hiperuricemia (14.5\%), hiperlipidemia (9.2\%) e obesidade (3.9\%) (Figura 5).

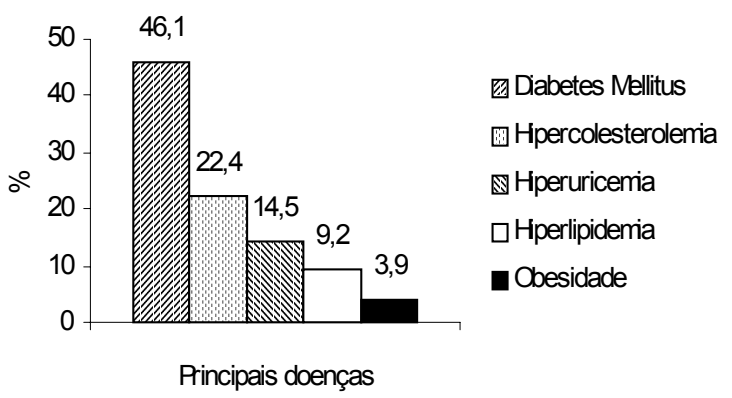

Figura 5 - Distribuição percentual dos diagnósticos mais freqüentes entre as causas endócrinometabólicas

Entre as causas mentais-comportamentais as principais doenças foram o transtorno neurovegetativo $(45.9 \%)$, depressão $(16.2 \%)$, ansiedade $(8.1 \%)$, stress (5.4\%) (Figura 6).

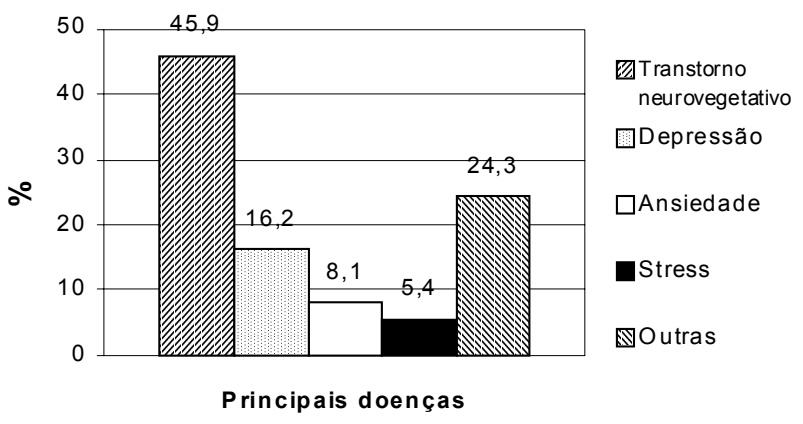

Figura 6 - Distribuição percentual dos diagnósticos mais freqüentes entre as causas mentaiscomportamentais

Ao se proceder o cruzamento para algumas variáveis sócio-demográficas, para cada uma destas três principais causas de morbidade no grupo de doenças crônico-degenerativas, observou-se que existe um predomínio das mulheres brancas e provenientes da zona urbana, provavelmente devido ao vício de seleção decorrente das características de atenção à saúde da unidade.

\section{DISCUSSÃO}

Os resultados deste estudo apontaram para uma demanda onde predominam os indivíduos do sexo feminino principalmente em idade fértil, sendo provavelmente esta a razão pela qual aparecem com um maior destaque as doenças ginecológicas e obstétricas. Isto denota que esta unidade tem se caracterizado essencialmente pelos moldes tradicionais de atendimento de unidades básicas de saúde que priorizam a saúde materno-infantil.

Observou-se que existe uma evasão dos indivíduos do sexo masculino na idade acima de 20 anos e, para ambos os sexos, nas idades acima de 40 anos, podendo-se constatar a necessidade de direcionamento de ações de saúde voltadas para estas faixas etárias, sem distinção de sexo, por ser este o papel de um serviço de atenção primária à saúde que contemple o verdadeiro perfil demográfico de sua população de referência.

Considerou-se, com base no exposto, que ao se pensar em atendimento primário à saúde, não seria viável o investimento somente no planejamento de ações que priorizem uma clientela composta por crianças e mulheres.

O perfil epidemiológico de morbidade desta unidade mostra que existe uma complexidade das questões que estão relacionadas à saúde destes indivíduos, se considerarmos que as doenças do aparelho cárdiovascular, endócrino-metabólico e comportamentais requerem um amplo planejamento de ações voltadas para as mesmas.

Dentro do grupo de doenças crônicodegenerativas destaca-se a necessidade de investimento em ações basicamente de prevenção e promoção à saúde, uma vez que a hipertensão arterial, o diabetes mellitus e as doenças comportamentais têm sido relevantes no perfil de morbidade desta unidade. Com base nestas considerações, parece oportuna a implementação, nesta unidade básica, de programas de reeducação alimentar, atividade física, controle do stress e auto-cuidado. Cabe salientar ainda que a hipertensão arterial e o diabetes mellitus constituem-se em fatores de risco para outras doenças crônicas como as cardiovasculares (MORAES \& SOUZA, $1996 \mathrm{a}, \mathrm{b})$.

As doenças infecciosas aparecem também com uma certa importância, principalmente as relacionadas ao aparelho respiratório, indicando que o perfil de morbidade desta unidade assemelha-se ao modelo polarizado de transição descrito por FRENK et al. (1991), onde as doenças crônico-degenerativas coexistem com as doenças infecto-parasitárias.

O processo histórico pelo qual o Brasil vêm passando, marcadamente presente no estado de São Paulo, caracterizado pela transição demográfica (envelhecimento populacional) e transição epidemiológica (aumento da morbi-mortalidade pelas doenças crônico-degenerativas), foi detectado também na demanda que compõe esta unidade básica de saúde, sugerindo a necessidade de que estes serviços se adeqüem às transformações que têm ocorrido no âmbito da dinâmica populacional do Brasil com suas conseqüentes implicações sobre o processo saúde-doença destas populações. 
DEMAND FOR CHRONIC-DEGENERATIVE DISEASES AMONG ADULTS ATTENDED IN A BASIC HEALTH UNIT AT THE CITY OF SÃO CARLOS-SP

The purpose of this study was to find out the profile of the larger clientele more than 12 years old of a Basic Health Unit (BHU), according to socio-demographic variables such as sex, age group, color, marital status, origin and to characterize the diseases profile according to the chapters of the Internacional Classification of Diseases (ICD X-Revision) and group specific diagnoses standing out the group of the chronic-degenerative diseases, searching for its its characterization in the context of the demographic-epidemiology transition. The study was of the transversal type and the population was constituted by the clientele registered in the BHU until August 31, 1996. Individuals in situation of abandonment, the ones tansferred to another units and those who died were excluded. The population studied was formed by 1013 individuals. Data were processed in the software FOXPRO vs 2.0 and the analysis developed in EPIINFO vs 6.04. Authors verified that the studied population was basically formed by women (87.1\%). Regarding age, 69.6\% were between 12 and 40 years old, 95.6\% were of white color, 57.2\% were married and $97 \%$ coming from the urban zone. The classification according to ICD chapters showed disturbances of the urinary tract and of the genital system (35.5\%), breathing system (11.5\%) and symptons and signs (9.9\%). The classification according to specific groups of causes showed similar proportions among the infect-parasitic diseases (24.8\%) and the chronic-degenerative diseases (24.5\%). In the group of chronic-degenerative diseases $50 \%$ of ICD chapters were formed by the groups: circulatory, endocrine causes and mental-behaviour. Authors observed that the profile of diseases at the BHU resembles the polarized model of transition where the chronic-degenerative diseases coexist with the infect-parasitic ones. The traditional model of care of the unit that focuses the maternal infant health seems not to adapt to the population progressive aging when prioritizing a clientele that although still young need collective actions for primary and secondary prevention of the chronic-degenerative diseases.

KEY WORDS: proportional morbidity, chronic-degenerative diseases, primary health care

\section{OCURRENCIAS DE MALES CRÓNICO-DEGENERATIVOS ENTRE ADULTOS INSCRITOS EN UNA UNIDAD BÁSICA DE SALUD EN SÃO CARLOS-SP}

El estudio tuvo como objetivo trazar el perfil de los pacientes de más de 12 años de edad de una UBS, (Unidad Básica de Salud) de acuerdo con las variables sócio-demográficas como: sexo, edad, raza, estado civil y procedencia, y caracterizar el perfil de dolencias según los capitulos del CID (X-Revisión) y grupos diagnósticos especificos destacándose el grupo de los males crónico-degenerativos, con la intención de caracterizarlos en el contexto de la Transición demográfico-epidemiológico. El alineamiento fue del tipo transversal, siendo que la población base fue constituida por los pacientes inscritos en la UBS hasta el 31 de agosto de 1996. Fueron excluidos los individuos abandonados, los transferidos para otras unidades y aquellos que han muerto. La población efectivamente estudiada fue compuesta de 1013 individuos. Los datos fueron procesados no software FOXPRO vs 2.0 y los análisis fueron efectuados en el EPIINFO vs 6.04. Fue constatada que la población estudiada era constituida básicamente por mujeres (87.1\%). En relación a edad 69.6\% estaban entre 12 y 40 años, $95.6 \%$ eran blancos, $57.2 \%$ eran casados y 97\% provenientes del perímetro urbano. Cuando los diagnósticos fueron clasificados según los capitulos del CID se destacaron los males del aparato genital-urinário (35.5\%), aparato respiratorio (11.5\%) y síntomas y pré-avisos (9.9\%). La clasificación según los grupos especificos de causas mostró magnitud semejante entre los males infecto-parasitários (24.8\%) y los males crónico-degenerativos (24.5\%). En el grupo de males crónico-degenerativos $50 \%$ de los capitulos del CID fueron compuestos por el conjunto de: causas circulatorias, endocrinas y mentales-comportamentales. Fue observado que el perfil de dolencias de la UBS parece ubicarse en modelo polarizado de transición donde los males crónico-degenerativos conviven con las infecto-parasitarias. El modelo tradicional de atención de la unidad que focaliza la salud materno-infantil parece no estar adecuado al envejecimiento progresivo de la población al priorizar una clientela que aunque joven ya denota la necesidad de que se desenvuelvan acciones colectivas mirando hacia la prevención primaria y secundaria de los males crónico-degenerativos.

TÉRMINOS CLAVES: morbilidad proporcional, enfermedades crónico-degenerativas, atención primaria de salud

\section{REFERÊNCIAS BIBLIOGRÁFICAS}

\section{ALMEIDA, M.C.P. Políticas de saúde no Brasil na} década de 80: resumo histórico. Ribeirão Preto: Escola de Enfermagem de Ribeirão Preto, s.d. (mimeo).

\section{BANCO MUNDIAL. BRASIL. Novo desafio à}

saúde do adulto. Washington, D.C., 1991. Parte

1. 134 p. (Série de estudos do Banco Mundial sobre países). (Datilografado). 
03. BARRETO, M.L.; CARMO, E.H. Mudanças em padrões de morbimortalidade: conceitos e métodos. In: MONTEIRO, C. A. Velhos e novos males da saúde no Brasil. A evolução do país e de suas doenças. São Paulo: HUCITEC NUPENS/USP, 1995. p. 17-30.

04. DEAN, J. et al. Epiinfo - computer programs for Epidemiology. Atlanta: Division of Surveillance and Epidemiologics Studies, Epidemiology Programs Office, Center for Disease Control, 1996.

05. FOX HOLDINGS INC. Fox pro v. 2.0. Pat. Pend. American Edition, 1991.

06. FRENK, J. et al. La transición epidemiológica en América Latina. Bol. Of. Sanit. Panam., v. 111, n. 6 , p. 485-96, 1991.

07. INFORME EPIDEMIOLÓGICO DO SUS. Brasília: Centro Nacional de Epidemiologia-CENEPI, v. 1, n. 3, ago. 1992. 161 p.

08. KALACHE, A.; VERAS, R.P.; RAMOS, L.R. O envelhecimento da população mundial. Um desafio novo. Rev. Saúde Pública, São Paulo, v.21, n.3, p. 200-10, 1987.

09.LAURENTI, R. Transição demográfica e transição epidemiológica. In: CONGRESSO BRASILEIRO DE EPIDEMIOLOGIA, 1, Campinas, Anais. Rio de Janeiro:ABRASCO, 1990.

10. MENDES, E.V. As políticas de saúde no Brasil nos anos 80: a conformação da reforma sanitária e a construção da hegemonia do projeto neoliberal. In: . Distrito Sanitário - O processo social de mudança das práticas sanitárias do Sistema Único de Saúde. 2. ed. São Paulo: HUCITEC-ABRASCO, 1994. p. 19-91.

11. MORAES, S.A.; SOUZA, J.M.P. Diabetes mellitus e doença isquêmica do coração: estudo tipo casocontrole. Rev. Saúde Pública, v. 30, n. 4, p. 36471, 1996a.

12. MORAES, S.A; SOUZA, J.M.P. Diabetes mellitus e doença isquêmica do coração: comparação por sexo. Arq. Bras. Cardiol., v. 66, n. 2, p. 59-63, $1996 b$.
13. OMRAN, AR. The epidemiologic transition: a theory of epidemiology of population change. Milkbank Memorial Fund Quarterly, v. 49, n. 4, p. 50938, 1971.

14. OMRAM, AR. The epidemiologic transition in the Americas. Washington: PAHO-University of Maryland at College Park, 1996.

15. ORGANIZAÇÃO MUNDIAL DE SAÚDE. OMS. Manual de classificação estatística internacional de doenças e problemas relacionados à saúde. São Paulo: Centro da OMS para classificação de doenças em português- USP, 1995.

16. PRATA, P.R. A transição epidemiológica no Brasil. Cad. Saúde Pública, Rio de Janeiro, v. 8, n. 2, p. 168-75, abr/jun.1992.

17. RAMOS, L.R.; VERAS, R.P.; KALACHE, A. Envelhecimento populacional: uma realidade brasileira. Rev. Saúde Pública, São Paulo, v. 21, n. 3, p. 211- 24, 1987.

18. RAMOS, L.R. et al. Perfil do idoso em área metropolitana na região Sudeste do Brasil: resultados de inquérito domiciliar. Rev. Saúde Pública, São Paulo, v. 27, n. 2, p. 87-94, 1993.

19. RIBEIRÃO PRETO (Cidade). Secretaria Municipal de Saúde. SICAEV- Sistema de coleta e análise de estatísticas vitais- mortalidade de residentes e não residentes em Ribeirão Preto. São Paulo, Boletim, v. 3, n.1, 1993.

20. SÃO PAULO (Cidade). Prefeitura Municipal. PROAIM- Programa de aprimoramento das informações de mortalidade no município de São Paulo. São Paulo, 1993. v. 12.

21. VERAS, R.P. Considerações acerca de um jovem país que envelhece. Cad. Saúde Pública, Rio de Janeiro, v. 4, n. 4, p. 382-97, out./dez. 1988.

22. VERAS, R.P.; RAMOS, L.R.; KALACHE, A. Crescimento da população idosa no Brasil: transformações e conseqüências na sociedade. Rev. Saúde Pública, São Paulo, v. 21, n. 3, p. 225-33, 1987. 